\title{
Full Mouth Rehabilitation with Tooth and Implant Supported Combination Prosthesis- A Case Report with 10 Years Follow-up
}

\author{
Pramod Kumar AV ${ }^{1}$, Vinni $\mathrm{TK}^{2}$, SameeraK $^{3}$, Mohamed Saheer K \\ 1. Associate Professor, ${ }^{2}$ Associate Professor, ${ }^{3}$ Junior Resident, 4 Assistant Professor \\ Department of Prosthodontics, Government Dental College Kozhikode, Calicut, India
}

\begin{abstract}
Problems can develop when teeth and implants are combined in the same prosthesis due to the difference in mobility of the two. However, by combining dental implants and natural teeth with the help of attachments, clinicians can greatly improve retention, stability and aesthetics of these prostheses. The biomechanical principles should be judiciously incorporated in the design to nullify the deleterious leverages exerted by the prosthesis and to equalize the stress exerted by the prosthesis on implant and teeth.
\end{abstract}

Keywords: Implants, Attachments, Combination prosthesis, Implantoverdenture, Full mouth rehabilitation.

\section{Introduction}

The high degree of success of dental implants has conquered the minds of clinicians and it has become increasinglycommon to use implants for the rehabilitation of partially or completely edentulous patients. The prosthesis fabricated over osseointegrated implants can be of two types:a removable overdenture or a fully anchored fixed prosthesis.Implant overdenture varies from simple ball-attachments to complexparallel milled bar prosthesis[1].

The implant overdentureprovides many practical advantages over the implant supported fixed prosthesis. They provide cost effective treatment modality with better support and function. Aesthetics can be enhanced byincorporating labial flangesfor soft tissue support. Hygiene conditions and home maintenance procedures are improved. Moreover the prosthesiscan be removed at night to reduce the effects of nocturnal parafunction and stresses on the implant support system. It provides stress relief between the superstructure and the prosthesis by allowing sharing of occlusal load with the soft tissue. The prosthesis is also easier to repair than fixed restorations. Thus shorter restorative appointments reduced laboratory fees and fewer implants allow the rehabilitation at easewhen compared to fixed prostheses [1-4].

Implant overdentures can be modified to incorporate natural teeth as supporting elements for providing additional retention, proprioception and support [5].There exists a debate as to whether implants should be splinted to natural teeth and if so, what design of linking should be used, due to difference in mobility [6].The difference in mobility can be of the order of $50-200 \mu \mathrm{m}$ for a healthy periodontal ligament while an integrated implant will have mobility of only $10 \mu \mathrm{m}$ [7]. Hence it is not advisable for a tooth to be rigidly connected to a fixed dental prosthesis that is supported by one or more implants [8][9].

Overdentures can be fabricated over multiple implants or natural teeth with or without splinting. The added natural teeth result in more retentive and supporting units which reduce stress on implants. As a result, complications are minimized and implant and prosthesis longevity are increased at a reduced treatment cost [10].

\section{Case Report}

A 52 year old male reported for replacement of missing teeth. Onexamination, there were only a few natural teeth present in the oral cavity of which most of them were periodontallycompromised. The teeth present were $17,16,13,12,22,26$ in the maxilla and 35,34,43,44 and 45 in the mandible(Fig. 1). The periodontal condition of 17, 13, 35, 34 and 44 were found to be satisfactory (Fig. 2). The systemic condition of the patient was clinically satisfactory.

Patient was informed about the need for extraction of the mobile teeth. The advantages and disadvantages of various prosthetic treatment modalities were explained to the patient. Treatment options were upper and lower implant fixed prosthesis, a tooth and implant supported overdentureor a conventional complete denture, of which an implant and tooth supported removable prosthesiswas recommended to get adequate soft tissue support. It was planned to retain the periodontallysound teeth and to extract the remaining followed by implant placement and fabrication of maxillary-mandibular implant and tooth supported overdenture.

Immediate placement of implants (UniTi, Equinox,Holland)wasdone in the 14, 12, 11, 22, 24 region in the maxilla and 33,31, 41, 42 region in the mandible.Endodontic treatment was completed for 17, 13, 35, 34 and 44.The prosthetic phase was initiated three months after placement of the implants. The natural teeth were 
sectioned $1 \mathrm{~mm}$ supragingivally and the root canals were prepared to receive overdentureprimary copings (Fig. $3)$.

An impression of the teeth and implants was then made in polyether (Impregum,3M ESPE) with custom fabricated tray made of autopolymerising acrylic resin using indirect transfer technique (Fig. 4).Casts were prepared in Type IV gypsum(Ultrarock,Kalabhai,India).A tentative jaw relation record was taken and casts were articulated in mean value articulator. Implant abutments were selected to get a common path of insertion and height of the abutments was adjusted. All abutments were milled at 3 degree taper with a milling machine (S3 Junior, Schick, Germany) (Fig. 5)(Fig.6).

Primary copings were fabricated in wax over the implant abutments and on prepared teeth. The primary copings of implants were splinted together to form a bar. Plastic castable attachments(Rhein 83 srl,Italy)were attached to bar and copings in a parallel manner, with the help of amilling machine and a paralleling tool (Fig.7). A screw retained Hader bar was fabricated in maxillary anterior region. The milled bar and copings were cast in chrome-cobalt alloy(Wironium plus,Bego,Germany)and polished to high gloss(Fig.8).After verifying the clinical fit, a pick-up impression was made with additional silicone in a stock tray, using putty wash single impression technique and the cast was poured in type IV gypsum(Fig.9).

Mandibular metal framework was fabricated by connecting castableplastic housings (Rhein 83srl,Italy) with pattern resin(GC,Japan), which was removed, invested and cast with chrome-cobalt(Fig. 10). In the maxillary arch, a secondary framework was fabricated by using refractory cast technique. After blocking out the undercuts with blockout wax, nylon retentive caps (Rhein $83 \mathrm{srl}$, Italy) were placed over the attachments. Spacer wax of $0.5 \mathrm{~mm}$ was placed over the saddle area. Casts were duplicated with agar and a refractory cast was made with phosphate bonded investment (Wirovest, Bego, Germany) (Fig.11) (Fig.12). Wax pattern for secondary framework was made (Fig.13)and cast in chrome-cobalt using conventional casting technique (Fig. 14). Facebow transfer was done along with bite registration by using bite rims prepared on secondary frameworkand articulated onasemi adjustable articulator.Teeth arrangement was done to establish bilateral balanced occlusion. After try in, denture was acrylised in heat cure acrylic resin (Fig.15).

During the insertion stage, primary copings were cemented with Type I Glass Ionomer cement (Fuji I, GC, Japan)(Fig. 16). Maxillary bar was secured by tightening abutment screws to $25 \mathrm{Ncm}$ pressure..

Nylon caps were inserted in the corresponding slots in the denture and the fit was checkedintraorally .Occlusion was verified and occlusal adjustments were done as required.

Oral hygiene instructions were given and patient was asked to report after 1 week for review. Recallvisits were scheduled afteronemonth,three months, followed by every six months. During each visit, the oral hygiene maintenance, periodontal health status and fit of the prostheses were assessed.The patient was satisfied with aesthetics, function and comfort and the case was followed up to ten years withradiographs (Fig. 17)(Fig. 18)(Fig. 19).

A ten year follow-up showed missing coping on 45 and mobility of 17 . The post space of 45 was sealed with amalgamand 17 was extracted later.All implants are now stable and the patient is using the overdenturecomfortably (Fig.20) (Fig.21).

\section{Discussion}

Tooth-implant combination prosthesis is one which is supported by both natural teeth and implants. In some cases, the distribution of the remaining natural teeth may not be favourable for fabrication of tooth supported overdentures, thus they should be supplemented with implants in certain areas.Even though they provide numerous advantages like reduced cost,proprioception,additional support etc.,the potential complications associated with them should also be analysed and considered.

The risk arises due to the difference in mobility and distribution of force between tooth / implant and bone.The periodontal ligament transmits forces to all the surrounding bone but implants concentrate the force at the crestal bone.But splinting of implants can be done as splinted implants provide better load sharing[11][12].

In a combined prosthesis, supported by both teeth and implants, the method of attachment between the segments can be flexible (over denture) or stiff (fixed prosthesis). When a lateral force is applied to the implants of the combined fixedprosthesis, no force is distributed to the teeth, but rather is concentrated at the implants as distractive long leverage arm[13].

In a combined overdenture, where there isa flexible connection between the overdenture and primary structures, the force applied to the teethwill not be transmitted to the implants in a deleterious manner as there is a stress breakage at the attachment level.Overdentures should be designed in such a way that they do not transmit lateral forces to the primary structures. In addition to this, stress equalisation between natural teeth and implants can be achieved by utilizing nylon caps of varying resiliency. 


\section{Conclusion}

A combined prosthesis is one which is supported by implants and natural teeth in the same arch. The distribution of forces in implant and natural tooth varies. Therefore the prosthesis should be designed not to concentrate stress at any points. By proper designing and execution of the prosthesis the problems associated with stress distribution can be overcome.

\section{Clinical significance}

The practise of extraction and replacement with implants has increased tremendously among the clinicians nowadays. As a result many teeth gets extracted and replaced with implants even in doubtful indications. Isolated teeth which are periodontally sound can be retained and incorporated in implant supported removable prosthesis without any complications.

\section{References}

[1] Inan O, Aykent F, Alptekin N. Implant supported overdenture therapy: a 3- to 8-year prospective study. Implant Dent. 2000; 9(4):369-73.

[2] Hobo S, Ichida E, Gacia LT. Osseointegration and occlusal rehabilitation. Tokyo: Quintessence; 1989. p. 187-208.

[3] Carl E Misch. Contemporary Implant Dentistry. $3^{\text {rd }}$ ed. Elsevier; 2007.p. 43-50.

[4] Watson CJ,OgdenAR,Tinsley D.A 3-6 year study of overdentures supported byhydroxyapatite coated endosseous dental implants.Int J Prosthodont.1998 Nov-Dec;11(6):610-9.

[5] Lang N,PjeturssonB, Tan K.A systematic review of the survival and complication rates of fixed partial dentures after an observation period of atleast 5 years,combined tooth implant supported FPDs.Clin Oral Implants Res.2004Dec;15(6):643-53.

[6] Davies SJ, Gray RJ, Young MPJ. Good occlusal practice in the provision of implant borne prostheses. Br Dental J. 2002Jan26;192(2):79-88.

[7] BrunskiJB,Puleo DA, Nanci A. Biomaterials andBiomechanics of oral/maxillofacial implants :Current status and future developments. Int J Oral Maxillofac Implants. 2000 Jan-Feb;15(1):15-46

[8] Palmer RM,Howe LC .Implant dentistry-prothodontics:a prospective 3 year study of fixed bridges linking Astra tech ST implants to natural teeth.Clin Oral Implants res.2005 Jun;16(3):302-7.

[9] El Charkawi Hussein G,ZerkyKhaled A, Mohammed T.Elwakad. Stress analysis of different osseointegrated implants supporting a distal extension prosthesis.JProsthet Dent 1994 Dec;72(6):614-22.

[10] PramodKumar A V,Vinni T K,Mahesh M R.Full mouth Rehabilitation with maxillary tooth supported and Mandibular tooth and implant supported combination prosthesis:A 4 year case report.J Indian Prosthodont Soc.2012 Jun;12(2):113-9.

[11] ReigerMR,MayberryM,BroseMO.Finite element analysis of a six endosseousimplants.JProsthet Dent.1990 Jun;63(6):671-6

[12] ClellandNL,IsmailYH,ZakiHS,PipkoD.Three dimensional finite element stress analysis in and around the screw and implant.Int $\mathrm{J}$ Oral Maxillifac Implants.1991 Winter;6(4):391-8.

[13] WienbergLA.Atlas of tooth and implant supported Prosthodontics.Quentessence Publishing Company;2003 .p. $51-52$

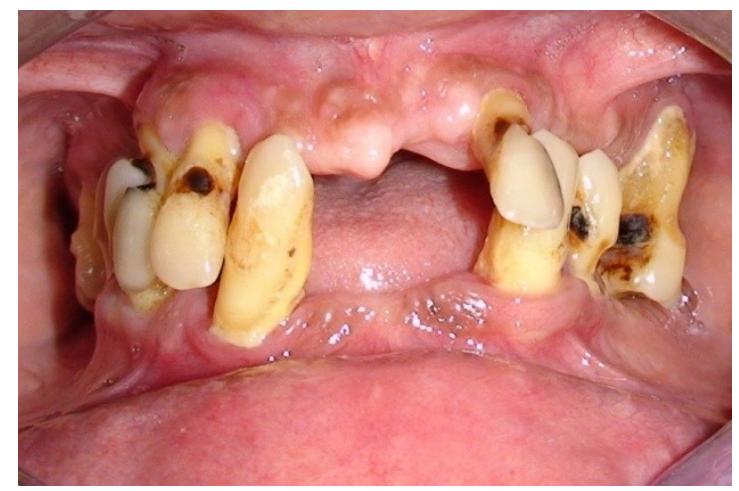

Figure 1.Preoperative photograph

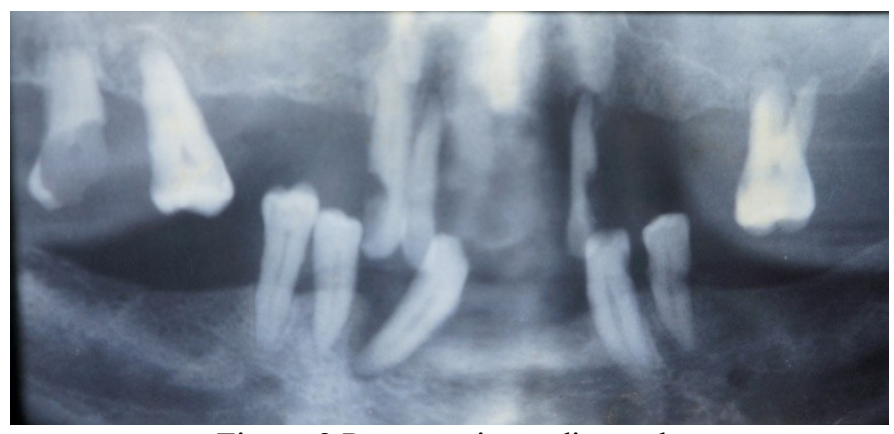

Figure 2.Preoperative radiograph 


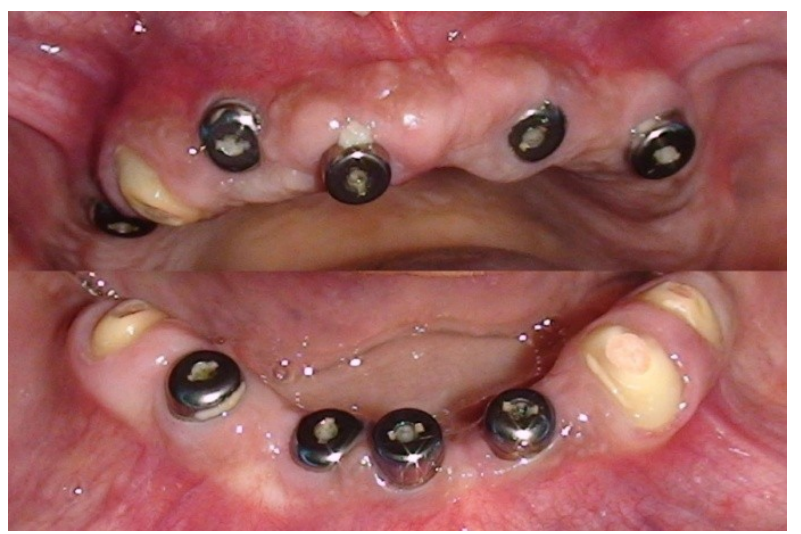

Figure 3.Implant per-mucosal extensions placed and tooth preparation done

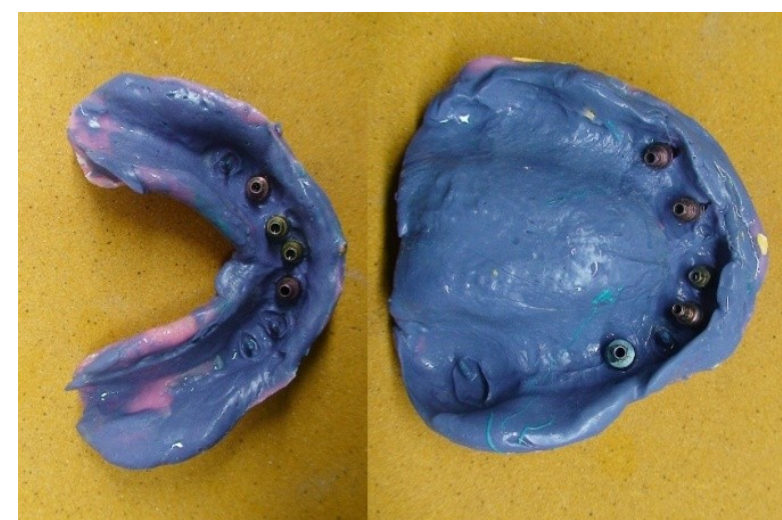

Figure 4.Upper and lower impressions

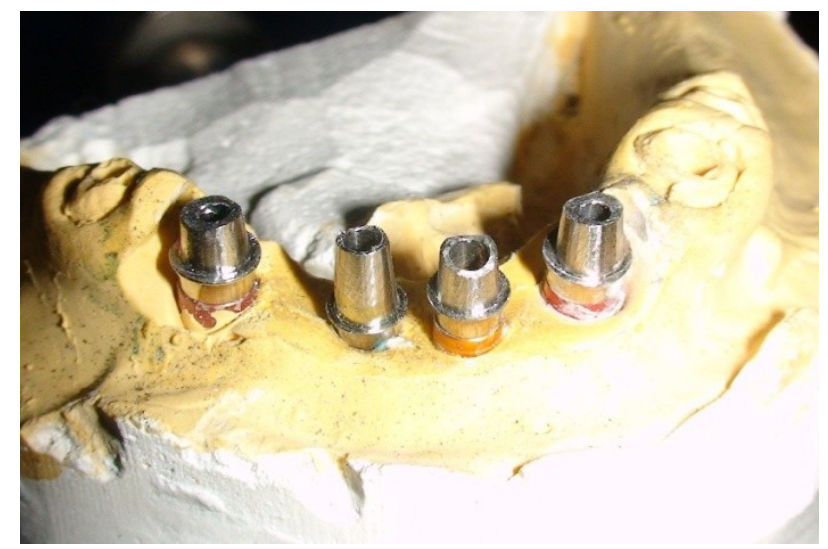

Figure 5.Abutments selected

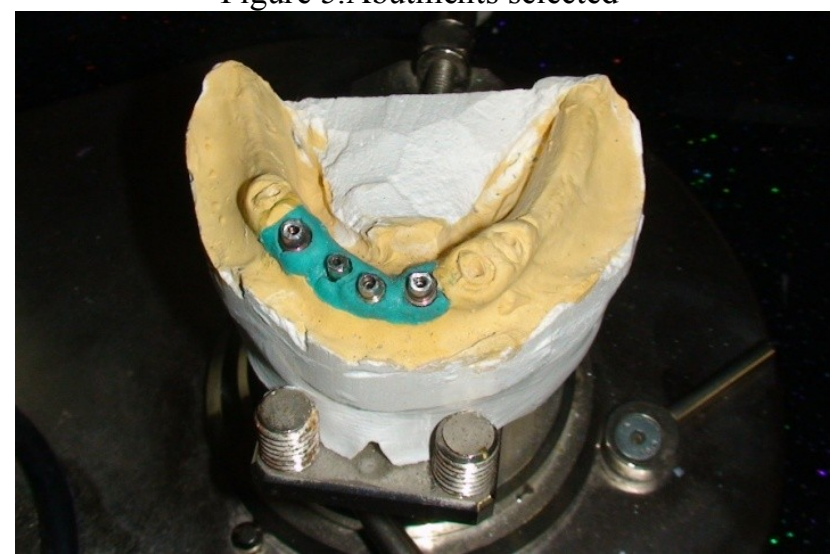

Figure 6.Abutments after milling 


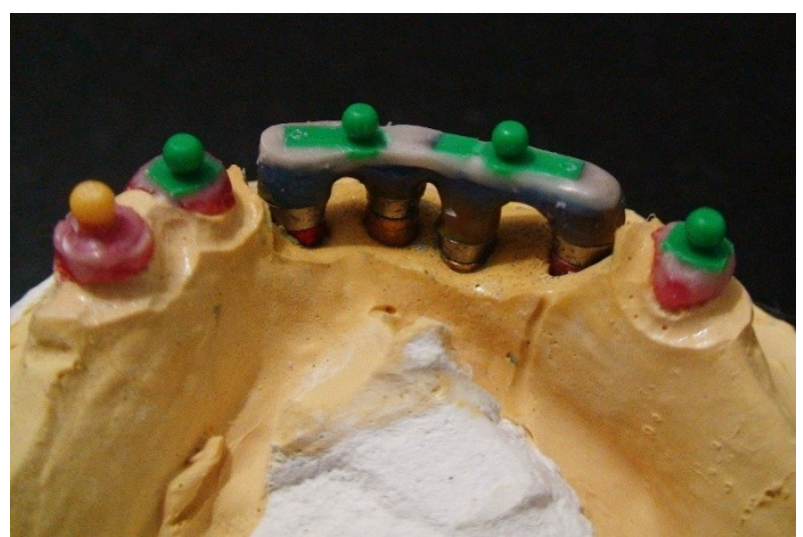

Figure 7.Primary copings with castable attachments

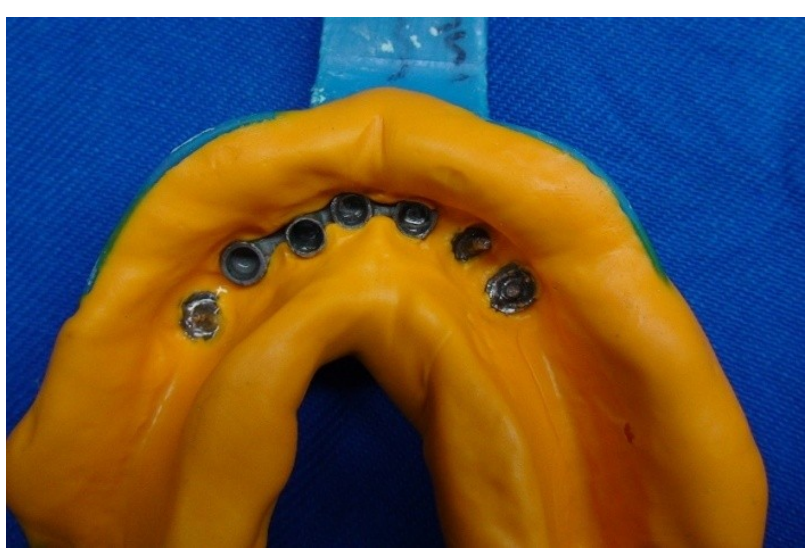

Figure 8.Finished primary coping

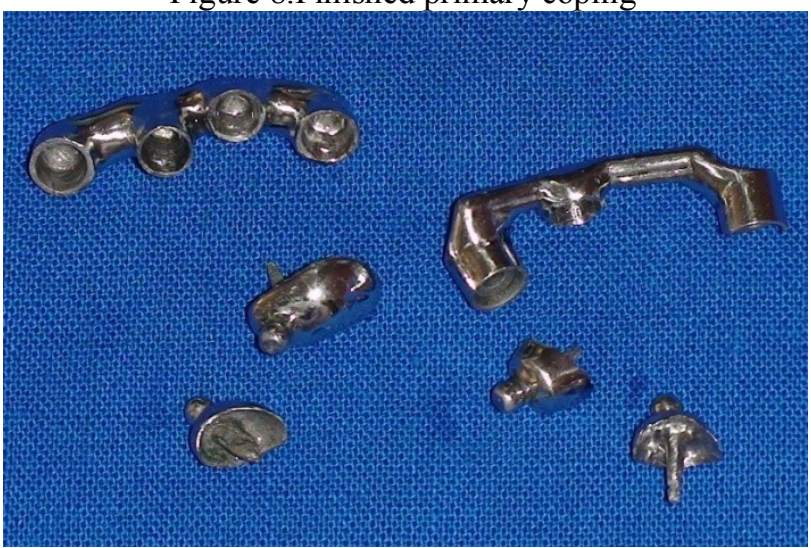

Figure 9.Pick-up impress

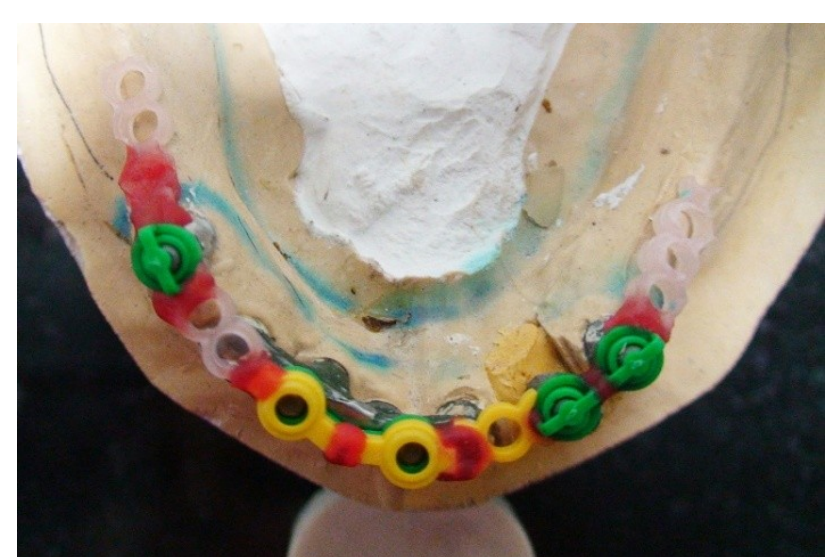

Figure 10.Plastic castable housings connected wiithpattern resin 


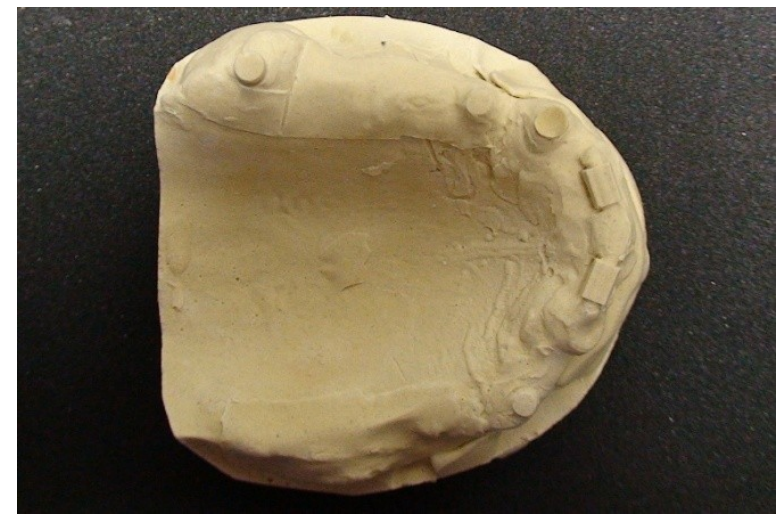

Figure 11.Maxillary cast ready to duplicate

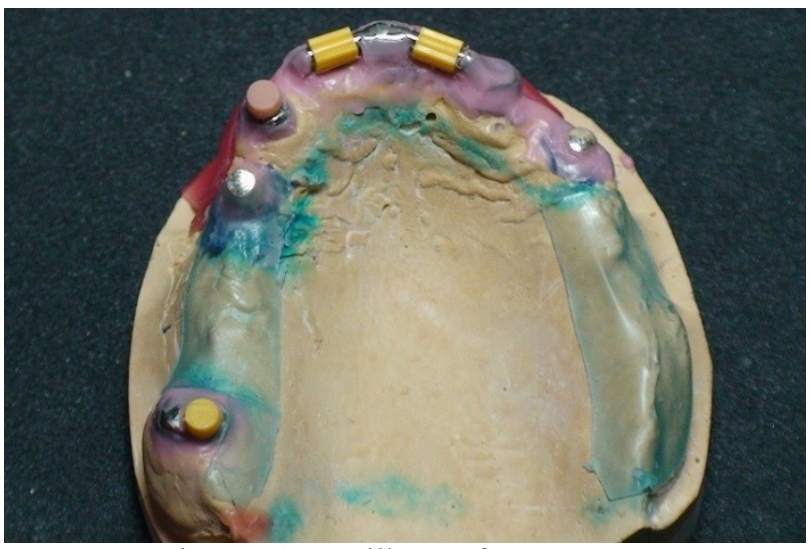

Figure 12.Maxillary refractory cast

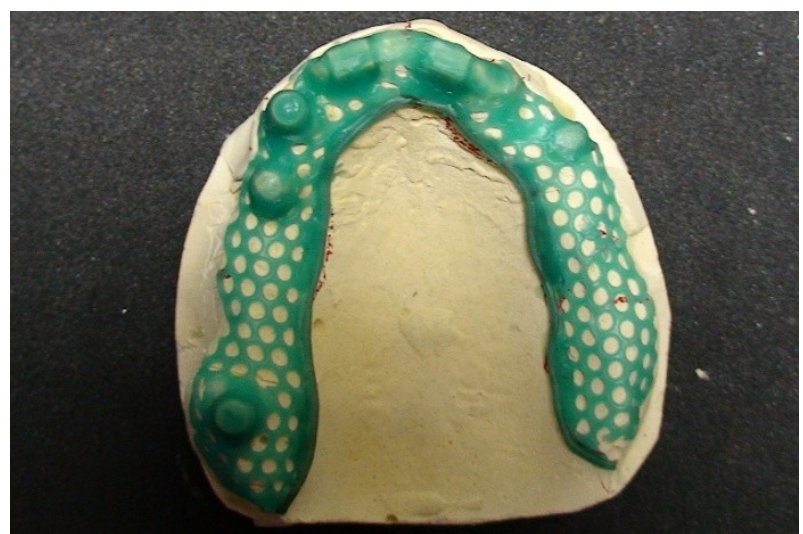

Figure 13.Maxillary secondary framework wax pattern

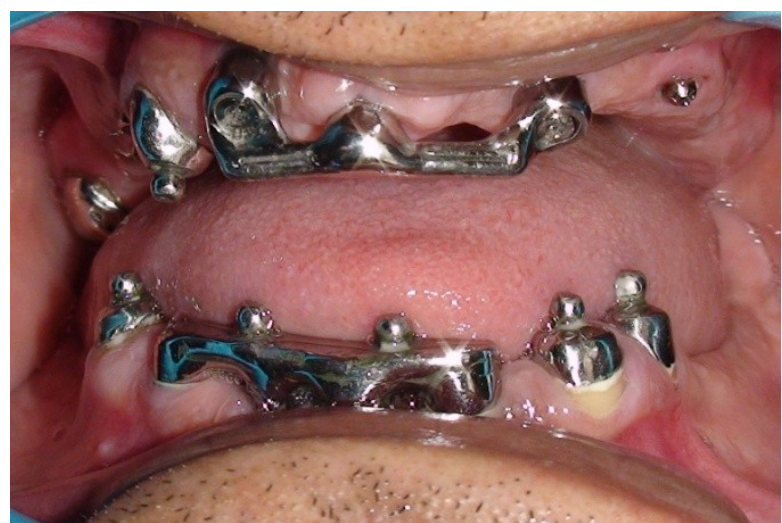

Figure 14.Upper and lower metal framework 


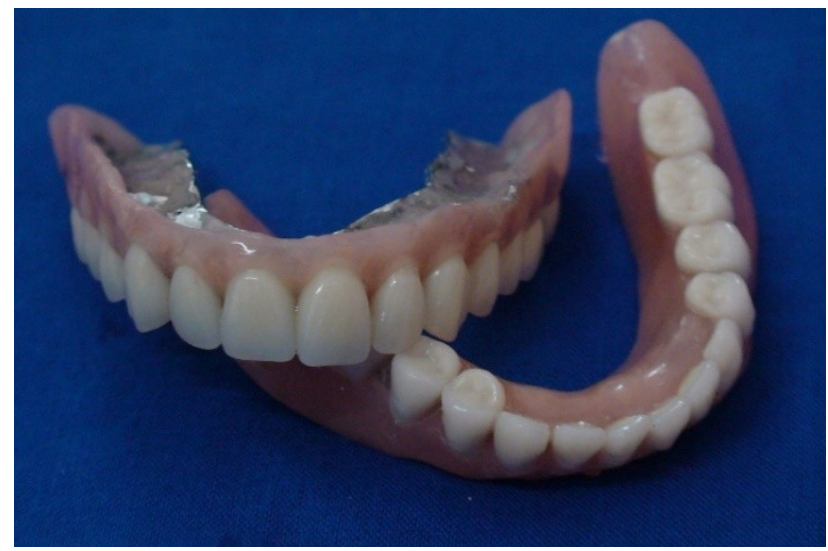

Figure 15.Finished dental prosthesis

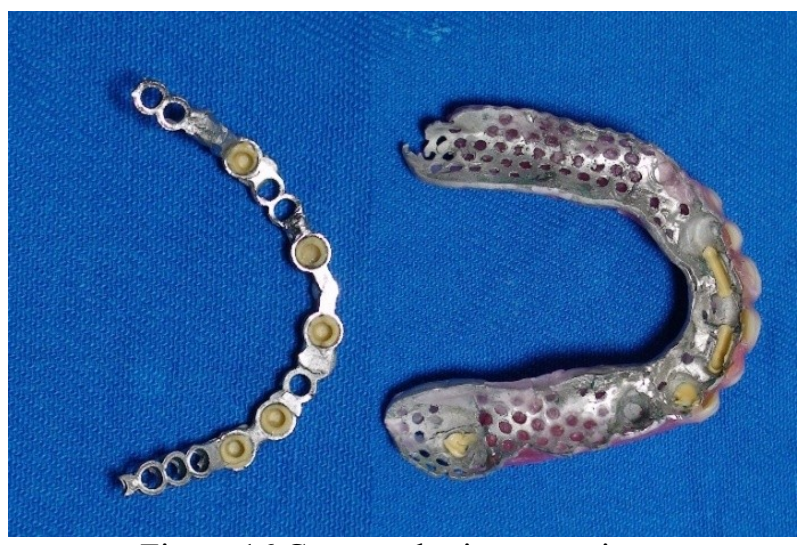

Figure 16.Cemented primary copings

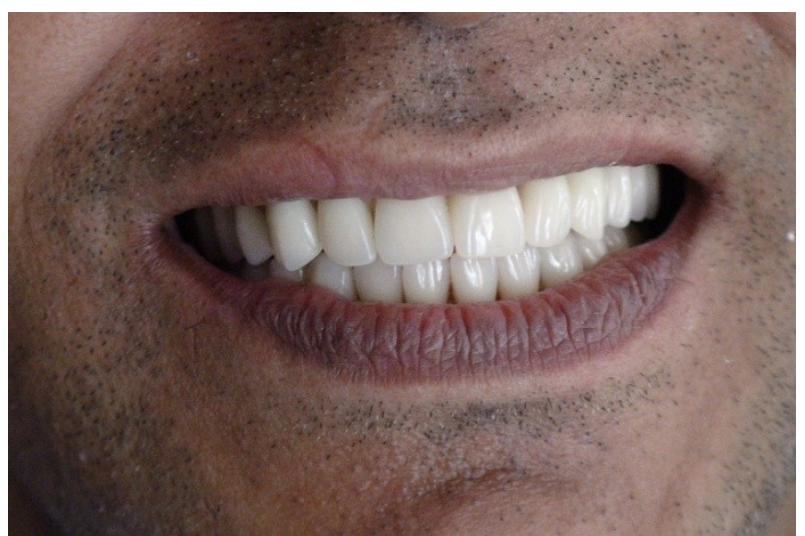

Figure 17.Immediate postoperative photograph

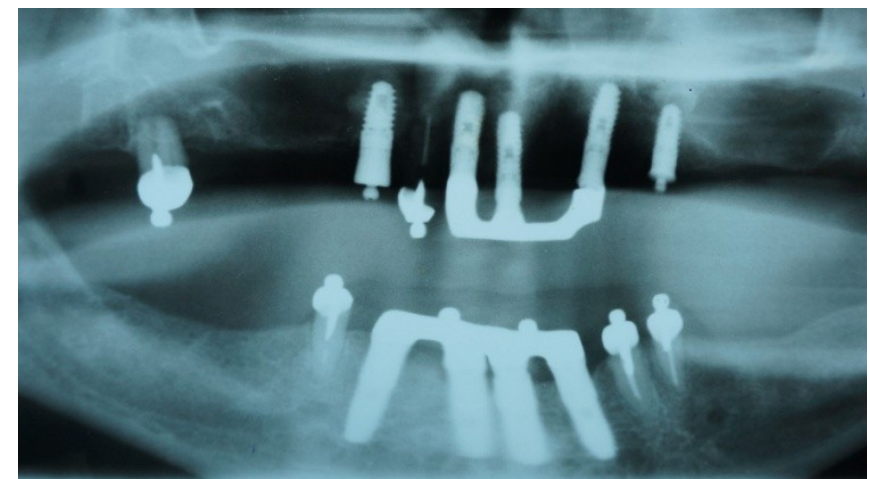

Figure 18.Immediate postoperative radiograph 


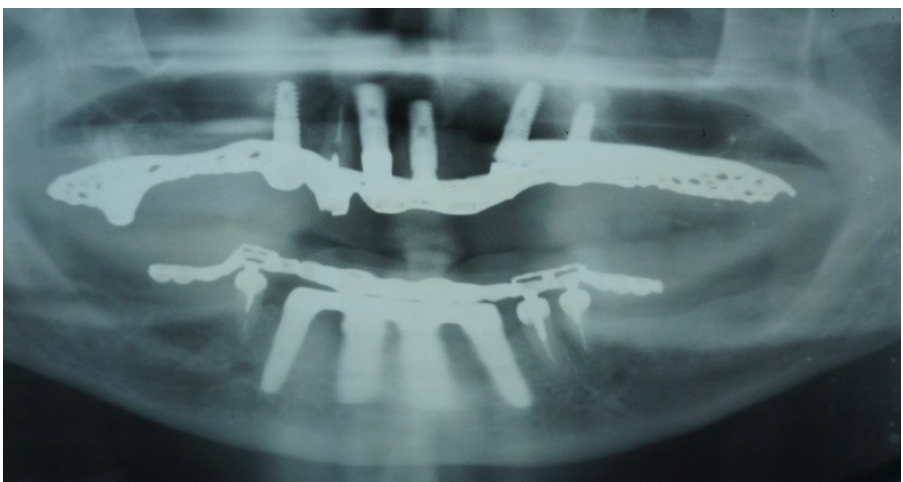

Figure 19.Radiograph after 6 years

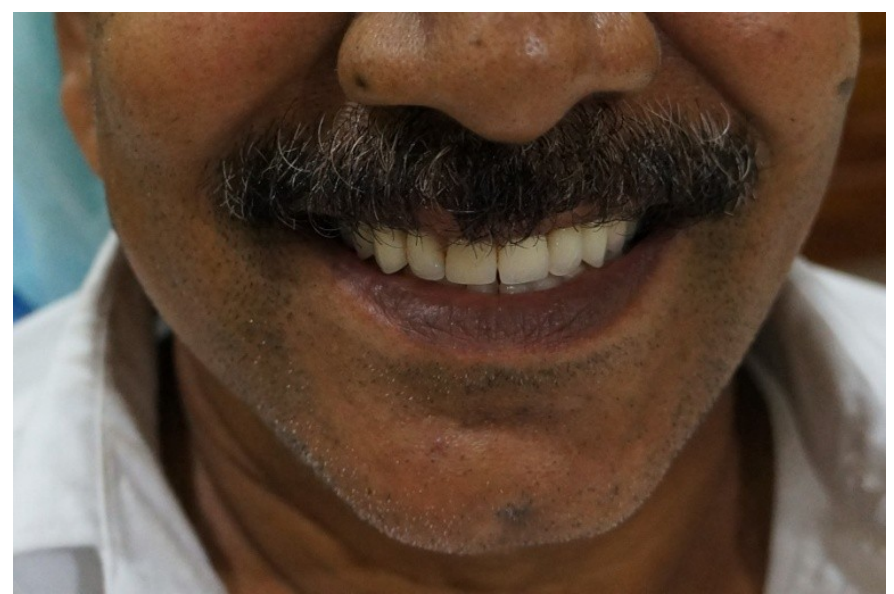

Figure 20.Postoperative photograph after 10 years

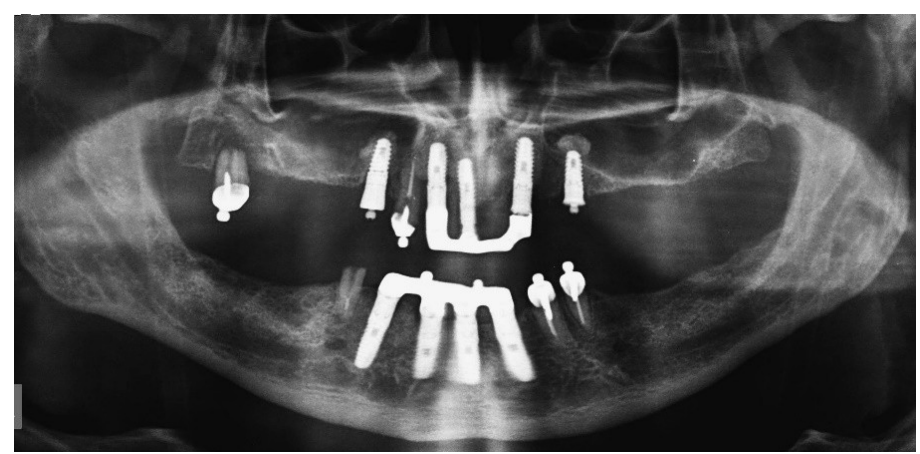

Figure 21.Postoperative radiograph after 10 years 